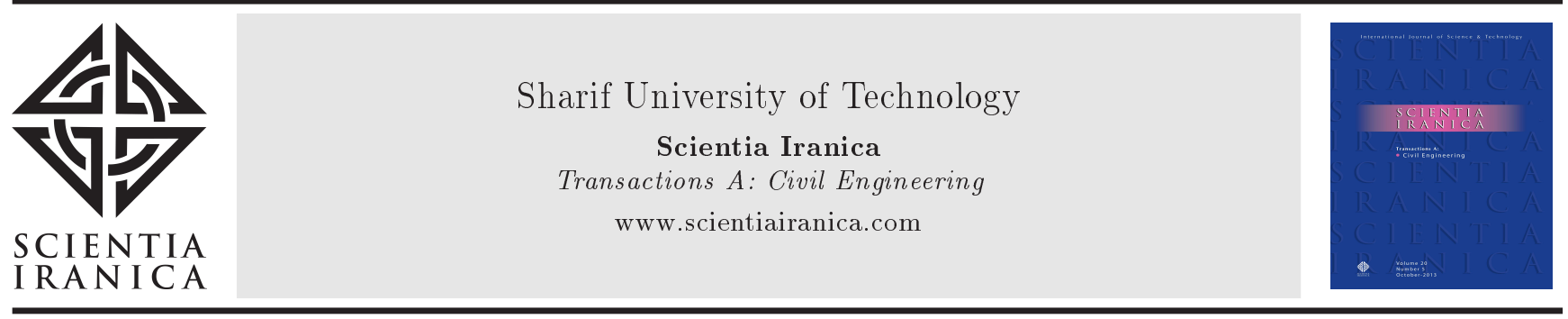

\title{
Three-dimensional axisymmetric responses of exponentially graded transversely isotropic tri-materials under interfacial loading
}

\author{
Y. Zafari ${ }^{\mathrm{a}}$, M. Shahmohamadi ${ }^{\mathrm{a}}$, A. Khojasteh ${ }^{\mathrm{b}, *}$ and M. Rahimian ${ }^{\mathrm{a}}$ \\ a. School of Civil Engineering, College of Engineering, University of Tehran, Tehran, P.O. Box 11155-4563, Iran. \\ b. School of Engineering Science, College of Engineering, University of Tehran, Tehran, P.O. Box 11155-4563, Iran. \\ Received 11 September 2015; received in revised form 29 January 2016; accepted 7 March 2016
}

\author{
KEYWORDS \\ Functionally graded \\ material; \\ Exponentially graded; \\ Transversely isotropic; \\ Displacement \\ potential; \\ Tri-material; \\ Axisymmetric green's \\ function.
}

\begin{abstract}
In this paper, an analytical formulation is presented to study an exponentially graded transversely isotropic tri-material under applied axisymmetric point-load and patchload with the aid of Hankel transform and use of a potential function. The given formulation is shown to be reducible to the special cases of (1) an inhomogeneous finite layer on a rigid base; (2) exponentially graded bi-material or half-space under applied buried loads; (3) homogeneous tri-material or bi-material solid. Several numerical solutions are presented to explain inhomogeneity effect on the stress transfer process in the inhomogeneous threelayered medium by means of a fast and accurate numerical method.
\end{abstract}

(C) 2017 Sharif University of Technology. All rights reserved.

\section{Introduction}

Functionally Graded Materials (FGM) with continuous variation of mechanical properties have extensive industrial applications such as thermal barriers, oxidation, and wear-resistant coatings due to improved residual stress distribution, thermal properties, and larger fracture toughness. Moreover, the better understanding of anisotropic and inhomogeneous characteristics of rock formations and naturally deposited layered soils is the reason for extensive studies of stress transfer in this type of solids with broad applications in geomechanics and foundation engineering.

For the continuum modelling of inhomogeneity in graded materials, different types of continuous property

\footnotetext{
*. Corresponding author. Tel.: +982161112232 E-mail addresses: yaserzafari@ut.ac.ir (Y. Zafari); mshahm@ut.ac.ir (M. Shahmohamadi); akhojasteh@ut.ac.ir (A. Khojasteh); rahimian@ut.ac.ir (M. Rahimian)
}

variations have been proposed including linear, exponential and power law, etc. [1].

Due to ease of formulation, for continuum modeling of FGMs, the exponential variation of the elastic constants is broadly used in numerical and analytical analyses. For a detailed review of studies in the field of anisotropic and nonhomogeneous isotropic materials, one might refer to [2]. The fundamental solutions concerning the exponentially graded elastic solids may be found in [3-8]. Esandari and Shodja [9] obtained fundamental solutions to an exponentially graded transversely isotropic half-space for arbitrary buried static loads.

Kashatalyan and Rushchitisky [10] presented two displacement potential functions to analyze a transversely isotropic inhomogeneous medium with functionally graded Young's and Shear moduli and constant Poisson's ratio. For the analysis of wave propagation in an inhomogeneous cross-anisotropic solid, one may refer to [11]. Also, Eskandari-Ghadi and AmiriHezaveh [12] studied the wave propagation problem 
in an exponentially graded transversely isotropic halfspace by presenting two potential functions. Selvadurai and Katebi [13] analyzed an incompressible elastic halfspace with the exponential variation of linear elastic shear modulus along the depth under vertical loads and studied the interaction of flexible and rigid plate with such media as [14-15].

Also, the contact analysis in graded materials is an interesting topic. Some of recent studies of this type may be found in [16-20], and the ReissnerSagoci problem for an arbitrary functionally graded solid in [21].

In the case of tri-materials, Kulchytsky-Zhyhailo and Bajkowski [22] studied a three-layered medium with two coating layers resting on half-space, where on the top layer, the circular normal and tangential loads are applied, and the interlayer is functionally graded by ensuring the continuous change of Young's modulus between the layers.

In this paper, with the aid of displacement potential functions presented by Eskandari-Ghadi and Amiri-Hezaveh [12], under axisymmetric condition, an exponentially graded transversely isotropic tri-material is analyzed for different types of vertical point, patch, and ring load patterns. Green's functions of stress and displacement components are given in terms of explicit line-integral representations. The results are verified to be in exact agreement with the solutions of inhomogeneous transversely isotropic half-space, homogeneous transversely isotropic tri-material fullspace, and incompressible inhomogeneous isotropic half-space $[9,23,13]$, respectively. Also, in the case of homogeneous transversely isotropic finite layer on a rigid base, the results are confirmed with the study by Small and Booker [24].

In this paper, the results may be utilized for the boundary integral equation formulation of geomechanical and foundation engineering problems and the interfacial fracture analysis in composite materials, or they may be used as benchmark solutions for the development of numerical methods for the analysis of inhomogeneous anisotropic materials.

\section{Statement of the problem and the governing equation}

With reference to Figure 1, a tri-material solid, consisting of a finite layer of arbitrary thickness $(h)$ perfectly bonded to two half-spaces, is considered for a cylindrical coordinate system $(r, \theta, z)$. All layers are assumed to be composed of different linearly elastic transversely isotropic materials where $z$-axis is the common axis of symmetry of materials, which is normal to the horizontal interfaces of the domains. Upper half-space $(z<0)$ is denoted as Layer I, middle finite layer $(0<z<h)$ as Layer II, and the lower half-

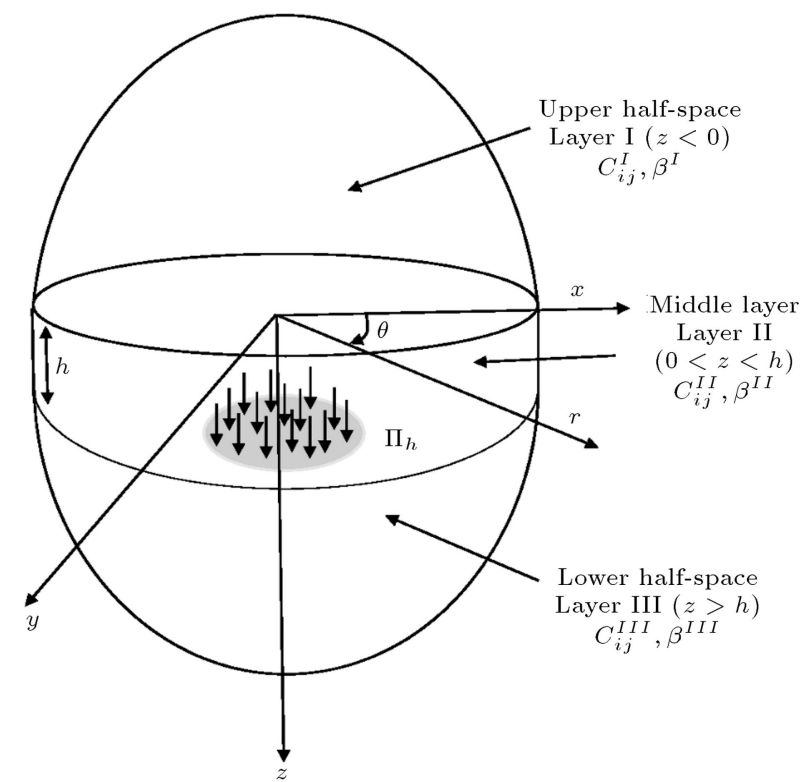

Figure 1. Functionally graded transversely isotropic tri-material under arbitrary interfacial vertical load.

space $(z>h)$ as Layer III. The elasticity constants of the layers are designated as $C_{i j}^{I}(z), C_{i j}^{I I}(z)$, and $C_{i j}^{I I I}(z)$, where the superscripts $I, I I$, and $I I I$ indicate the properties of Layers I, II, and III, respectively. The inhomogeneity is considered with the arbitrary exponential variation of material properties along $z$ axis in each layer. Therefore, the elasticity constants are expressed as:

in Layer I:

$$
c_{i j}^{I}(z)=C_{i j}^{I} e^{2 \beta^{I} z},
$$

in Layer II:

$$
c_{i j}^{I I}(z)=C_{i j}^{I I} e^{2 \beta^{I I} z},
$$

in Layer III:

$$
c_{i j}^{I I I}(z)=C_{i j}^{I I I} e^{2 \beta^{I I I} z},
$$

where $C_{i j}^{I}, C_{i j}^{I I}$, and $C_{i j}^{I I I}$ are the elasticity constants corresponding to depths $z=0^{-}, z=0^{+}$, and $z=h^{+}$, respectively, and relation $C_{66}=\left(C_{11}-C_{12}\right) / 2$ exists between the constants. Term $\beta$ is the exponential factor characterizing the alteration made to material properties along $z$ where $\beta=0$, representing a homogenous transversely isotropic tri-material.

For an exponentially graded transversely isotropic elastic material, in the absence of body forces and axisymmetric static condition, the governing equilibrium equations in terms of displacement components and cylindrical coordinate system may be expressed as: 


$$
\begin{aligned}
& C_{11}=\left(\frac{\partial^{2} u_{r}}{\partial r^{2}}+\frac{1}{r} \frac{\partial u_{r}}{\partial r}-\frac{u_{r}}{r^{2}}\right)+C_{44} \frac{\partial^{2} u_{r}}{\partial z^{2}} \\
&+\left(C_{13}+C_{44}\right) \frac{\partial^{2} u_{z}}{\partial r \partial z}+2 \beta C_{44}\left(\frac{\partial u_{r}}{\partial z}+\frac{\partial u_{z}}{\partial r}\right)=0 \\
& C_{44}\left(\frac{\partial^{2} u_{z}}{\partial r^{2}}+\frac{1}{r} \frac{\partial u_{z}}{\partial r}\right)+C_{33} \frac{\partial^{2} u_{z}}{\partial z^{2}} \\
&+\left(C_{13}+C_{44}\right)\left(\frac{\partial^{2} u_{r}}{\partial r \partial z}+\frac{1}{r} \frac{\partial u_{r}}{\partial z}\right) 2 \beta C_{33} \frac{\partial u_{z}}{\partial z} \\
&+2 \beta C_{13}\left(\frac{\partial u_{r}}{\partial r}+\frac{u_{r}}{r}\right)=0
\end{aligned}
$$

where $u_{r}$ and $u_{z}$ are the displacement components in radial $(r)$ and vertical $(z)$ directions, respectively. In order to uncouple the above equilibrium equations, displacement potential function $(F)$, introduced in [12], is used. This potential function is related to displacement components $u_{r}$ and $u_{z}$ as:

$$
\begin{aligned}
& u_{r}(r, z)=-\alpha_{3} \frac{\partial^{2} F(r, z)}{\partial r \partial z}-2 \alpha_{2} \beta \frac{\partial F(r, z)}{\partial r} \\
& u_{z}(r, z)=\left[\left(1+\alpha_{1}\right) \nabla_{r}^{2}+\alpha_{2} \frac{\partial}{\partial z}\left(2 \beta+\frac{\partial}{\partial z}\right)\right] F(r, z),
\end{aligned}
$$

where:

$$
\nabla_{r}^{2}=\frac{\partial^{2}}{\partial r^{2}}+\frac{1}{r} \frac{\partial}{\partial r}
$$

and:

$$
\begin{aligned}
& \alpha_{1}=\frac{C_{12}+C_{66}}{C_{66}}, \quad \alpha_{2}=\frac{C_{44}}{C_{66}}, \\
& \alpha_{3}=\frac{C_{13}+C_{44}}{C_{66}}, \quad \alpha_{4}=\frac{C_{33}}{C_{66}} .
\end{aligned}
$$

Putting Eq. (5) into Eq. (4) leads to a single partial differential equation as the governing equation for potential function, $F$ :

$$
\left[\nabla_{1}^{2} \nabla_{2}^{2}-4 \frac{\alpha_{3}-\alpha_{2}}{1+\alpha_{1}} \beta^{2} \nabla_{r}^{2}\right] F(r, z)=0
$$

where:

$$
\begin{aligned}
& \nabla_{i}^{2}=\nabla_{r}^{2}+\frac{1}{s_{i}^{2}}\left(\frac{\partial^{2}}{\partial z^{2}}\right)_{\beta}-\frac{\beta^{2}}{s_{i}^{2}}, \quad i=1,2 \\
& \left(\frac{\partial^{2}}{\partial z^{2}}\right)_{\beta} Y=\frac{\partial^{2}}{\partial z^{2}}\left(e^{\beta z} Y\right) .
\end{aligned}
$$

Here, $s_{1}$ and $s_{2}$ are the roots of the following equation and are not zero or pure imaginary numbers [25]:

$$
\alpha_{4} \alpha_{2} s^{4}+\left(\alpha_{3}^{2}-\alpha_{2}^{2}-\left(1+\alpha_{1}\right) \alpha_{4}\right) s^{2}+\alpha_{2}\left(1+\alpha_{1}\right)=0
$$

The zeroth-order Hankel transform is defined as [26]:

$$
\begin{aligned}
& \tilde{f}^{0}(\xi, z)=\int_{0}^{\infty} r f(r, z) J_{0}(r \xi) d r, \\
& f(r, z)=\int_{0}^{\infty} \widetilde{\xi f^{0}}(r, z) J_{0}(r, \xi) d \xi,
\end{aligned}
$$

where $J_{0}$ is the Bessel function of the first kind of order zero. By applying such a transform to Eq. (8) with respect to the radial coordinate, the following ordinary differential equation is obtained for $F$ :

$$
\left[\tilde{\nabla}_{1}^{20} \tilde{\nabla}_{2}^{20}+4 \frac{\alpha_{3}-\alpha_{2}}{1+\alpha_{1}} \beta^{2} \xi^{2}\right] \tilde{F}^{0}=0
$$

where:

$$
\tilde{\nabla}_{i}^{20}=-\xi^{2}+\frac{1}{s_{i}^{2}}\left(\frac{d^{2}}{d z^{2}}\right)_{\beta}-\frac{\beta^{2}}{s_{i}^{2}}, \quad i=1,2 .
$$

By means of Eq. (5) and the identities involving Hankel transforms, the transformed displacementpotential relations may be written compactly as:

$$
\begin{aligned}
& \tilde{u}_{r}^{1}=\alpha_{3} \xi \frac{\partial \tilde{F}^{0}(\xi, z)}{\partial z}+2 \alpha_{2} \beta \xi \tilde{F}^{0} \\
& \tilde{u}_{z}^{0}=\left[\alpha_{2} \frac{\partial}{\partial z}\left(2 \beta+\frac{\partial}{\partial z}\right)-\xi^{2}\left(1+\alpha_{1}\right)\right] \tilde{F}^{0} .
\end{aligned}
$$

The similar expressions for stress-potential functions may be expressed as:

$$
\begin{aligned}
& \tilde{\sigma}_{z r}^{1}= {\left[C_{44} \xi\left(\left(\alpha_{3}-\alpha_{2}\right) \frac{\partial^{2}}{\partial z^{2}}+\xi^{2}\left(1+\alpha_{1}\right)\right) \tilde{F}^{0}\right] e^{2 \beta z}, } \\
& \tilde{\sigma}_{z z}^{0}= {\left[C _ { 3 3 } \frac { \partial } { \partial z } \left(\alpha_{2} \frac{\partial}{\partial z}\left(2 \beta+\frac{\partial}{\partial z}\right)-\xi^{2}\left(1+\alpha_{1}\right)\right.\right.} \\
&\left.\left.+\alpha_{3} \frac{C_{13}}{C_{33}} \xi^{2}\right) \tilde{F}^{0}+2 \alpha_{2} \beta C_{13} \xi^{2} \tilde{F}^{0}\right] e^{2 \beta z}, \\
& \tilde{\sigma}_{r r}^{0}+2 C_{66} e^{2 \beta z}\left\{\left(\frac{\tilde{u}_{r}}{r}\right)^{0}\right\}=e^{2 \beta z}\left\{\frac { \partial } { \partial z } \left[\alpha_{3} C_{11} \xi^{2}\right.\right. \\
&\left.-C_{13} \xi^{2}\left(1+\alpha_{1}\right)+\alpha_{2} C_{13} \frac{\partial}{\partial z}\left(2 \beta+\frac{\partial}{\partial z}\right)\right] \\
&\left.+2 \alpha_{2} \beta C_{11} \xi^{2}\right\} \tilde{F}^{0}, \\
& \tilde{\sigma}_{\theta \theta}^{0}-2 C_{66} e^{2 \beta z}\left\{\left(\frac{\tilde{u}_{r}}{r}\right)^{0}\right\}=e^{2 \beta z}\left\{\frac { \partial } { \partial z } \left[\alpha_{3} C_{12} \xi^{2}\right.\right.
\end{aligned}
$$




$$
\begin{aligned}
& \left.-C_{13} \xi^{2}\left(1+\alpha_{1}\right)+\alpha_{2} C_{13} \frac{\partial}{\partial z}\left(2 \beta+\frac{\partial}{\partial z}\right)\right] \\
& \left.+2 \alpha_{2} \beta C_{12} \xi^{2}\right\} \tilde{F}^{0} .
\end{aligned}
$$

The general solution to Eq. (13) can be written as:

$$
\begin{aligned}
\tilde{F}^{0}(\xi, z)= & e^{-\beta z}\left[A(\xi) e^{\lambda_{1} z}+B(\xi) e^{-\lambda_{1} z}+C(\xi) e^{\lambda_{2} z}\right. \\
& \left.+D(\xi) e^{-\lambda_{2} z}\right]
\end{aligned}
$$

where constants $A, \ldots, D$ are determined by satisfying the boundary conditions. Also, $\lambda_{1}$ and $\lambda_{2}$ are obtained as:

$$
\begin{aligned}
& \lambda_{1}=\sqrt{a \xi^{2}+b+\frac{1}{2} \sqrt{c \xi^{4}+d \xi^{2}}}, \\
& \lambda_{2}=\sqrt{a \xi^{2}+b-\frac{1}{2} \sqrt{c \xi^{4}+d \xi^{2}}},
\end{aligned}
$$

where:

$$
\begin{aligned}
& a=\frac{1}{2}\left(s_{1}^{2}+s_{2}^{2}\right), \quad b=\beta^{2}, \\
& c=\left(s_{1}^{2}-s_{2}^{2}\right)^{2}, \quad d=-16 \frac{\alpha_{3}-\alpha_{2}}{\alpha_{4}} \beta^{2} .
\end{aligned}
$$

Consistent with the regularity condition at infinity, the general solution to $F, \mathrm{Eq}$. (17), can be rearranged as:

in Layer I:

$$
\tilde{F}^{0}(\xi, z)=e^{-\beta^{I} z}\left[A^{I}(\xi) e^{\lambda_{1}^{I} z}+C^{I}(\xi) e^{\lambda_{2}^{I} z}\right],
$$

in Layer II:

$$
\begin{aligned}
\tilde{F}^{0}(\xi, z)= & e^{-\beta^{I I} z}\left[A^{I I}(\xi) e^{\lambda_{1}^{I I} z}+B^{I I}(\xi) e^{-\lambda_{1}^{I I} z}\right. \\
& \left.+C^{I I}(\xi) e^{\lambda_{2}^{I I} z}+D^{I I}(\xi) e^{-\lambda_{2}^{I I} z}\right]
\end{aligned}
$$

in Layer III:

$$
\begin{aligned}
\tilde{F}^{0}(\xi, z)= & e^{-\beta^{I I I} z}\left[B^{I I I}(\xi) e^{-\lambda_{1}^{I I I} z}\right. \\
& \left.+D^{I I I}(\xi) e^{-\lambda_{2}^{I I I} z}\right] .
\end{aligned}
$$

Therefore, determination of the above 8 coefficients for the exponentially graded tri-material full-space problem of interest is required.

With the aid of equations Eqs. (15) and (16), the imposition interfacial stress and displacement compatibility conditions associated with a tri-material FGM under applied load are greatly facilitated, as illustrated in the following sections.

\section{Applying boundary conditions}

\subsection{Functionally graded tri-material under loading at $z=0$ or $z=h$}

An axisymmetric vertical load on subdomain $\Pi_{0}$ or $\Pi_{h}$, located at depth $z=0$ or $z=h$, respectively, may be written as a prescribed stress discontinuity:

$$
\begin{aligned}
& \sigma_{z z}\left(r, 0^{-}\right)-\sigma_{z z}\left(r, 0^{+}\right)= \begin{cases}-R(r), & r \in \Pi_{0} \\
0, & r \notin \Pi_{0}\end{cases} \\
& \sigma_{r z}\left(r, 0^{-}\right)=\sigma_{r z}\left(r, 0^{+}\right), \quad 0 \leq r \leq \infty,
\end{aligned}
$$

or:

$$
\begin{aligned}
& \sigma_{z z}\left(r, h^{-}\right)-\sigma_{z z}\left(r, h^{+}\right)= \begin{cases}-R(r), & r \in \Pi_{h} \\
0, & r \notin \Pi_{h}\end{cases} \\
& \sigma_{r z}\left(r, h^{-}\right)=\sigma_{r z}\left(r, h^{+}\right), \quad 0 \leq r \leq \infty,
\end{aligned}
$$

where $R(r)$ is the specified interfacial traction in vertical direction. Eight equations are obtained to find unknown coefficients $A^{I}, \ldots, D^{I I I}$ with respect to the aid of displacement, stress potential functions (Eqs. (15) and (16)) and Relations (20) to (22), stress discontinuity condition (Eq. (23)) along with the continuity of stresses, and displacements along $z=0$ and $z=h$. These equations are arranged in matrix form as:

$$
\begin{gathered}
I(\xi)\left[A^{I}(\xi) C^{I}(\xi) A^{I I}(\xi) B^{I I}(\xi) C^{I I}(\xi) D^{I I}(\xi)\right. \\
\left.B^{I I I}(\xi) D^{I I I}(\xi)\right]^{T}=S^{0}(\xi)
\end{gathered}
$$

where $I(\xi)$ is given in the Appendix, and $S^{0}(\xi)$ is the source vector described by:

$$
S^{0}(\xi)=\left[\begin{array}{llllllll}
0 & 0 & 0 & 0 & 0 & -R^{0}(\xi) & 0 & 0
\end{array}\right]^{T},
$$

for loading at $z=0$ and:

$$
S^{0}(\xi)=\left[\begin{array}{llllllll}
0 & 0 & 0 & 0 & 0 & 0 & 0 & -R^{0}(\xi)
\end{array}\right]^{T},
$$

for loading at $z=h$. The term $R^{0}(\xi)$ is the Hankel transformed function of the applied traction. Setting $\beta^{I}=\beta^{I I}=\beta^{I I}=0$, the above formulation degenerates exactly to the static analysis of an axially loaded homogeneous transversely isotropic tri-material by Khojasteh et al. [23].

\subsection{Functionally graded finite layer on a rigid base under surface loading at $z=0$}

In the previously described analysis, the formulation of an exponentially graded finite layer on a rigid base is obtained (see Figure 2) by removing Layer I, $C_{i j}^{I} \rightarrow 0$, 


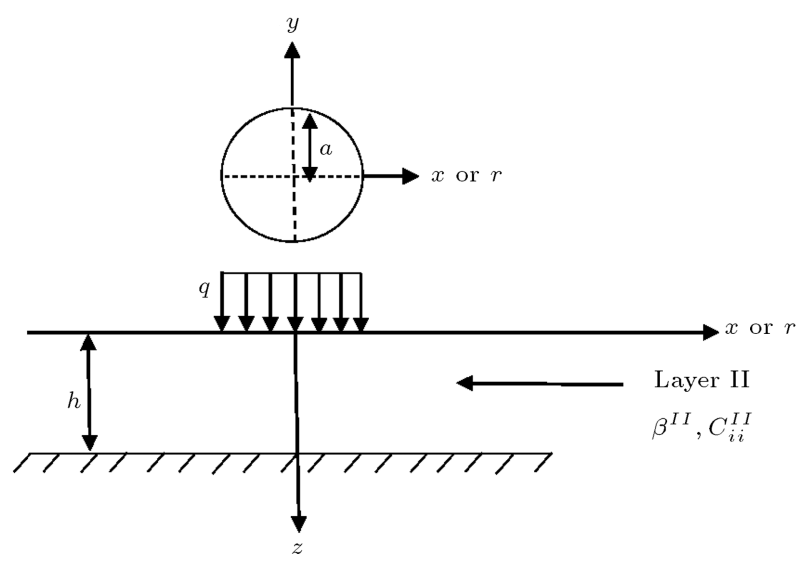

Figure 2. Functionally graded finite layer on a rigid half-space due under applied patch-load with a rough-rigid condition at interface.

applying load to the top of Layer II, and extremely increasing the elastic constants of Layer III to resemble a rigid half-space. The stress boundary conditions on the surface of layer, $z=0$, are described as:

$$
\begin{aligned}
& \sigma_{z r}(r, 0)=0 \\
& \sigma_{z z}(r, 0)= \begin{cases}-R(r), & r \in \Pi_{0} \\
0, & r \notin \Pi_{0}\end{cases}
\end{aligned}
$$

Here, the boundary conditions on the lower interface are assumed to be the rough-rigid or smooth-rigid [27] cases, defined as fully bonded or free sliding conditions, respectively.

The rough-rigid case which resembles the infinite friction capacity at the interface is defined as:

$$
u_{r}(r, h)=u_{z}(r, h)=0,
$$

and the smooth-rigid condition may be described as:

$$
u_{z}(r, h)=0, \quad \sigma_{z r}(r, h)=0 .
$$

By applying these boundary conditions, the matrix formation of the equations is given as:

$$
I(\xi)\left[A^{I I}(\xi) B^{I I}(\xi) C^{I I}(\xi) D^{I I}(\xi)\right]^{T}=S^{0}(\xi)
$$

where $I(\xi)$ is presented in Appendix A, and $S^{0}(\xi)$ is written as:

$$
S^{0}(\xi)=\left[\begin{array}{llll}
0 & -R^{0}(\xi) & 0 & 0
\end{array}\right]^{T} .
$$

\section{Axisymmetric Green's functions}

In this section, the axisymmetric Green's functions for different types of loading are given.

\subsection{Point-load}

A vertical point load with resultant $F_{v}$ and the relevant Hankel transformed loading coefficient can be written as:

$$
R(r)=\frac{F_{v} \delta(r)}{2 \pi r} \rightarrow R^{0}(\xi)=\frac{F_{v}}{2 \pi} .
$$

With the aid of inverse Hankel transform and using Eqs. (15) and (16), the displacement and stress responses of solid due to vertical point-load may be expressed as:

$$
\begin{aligned}
\hat{u}_{r}^{z}(r, z ; \beta)= & \int_{0}^{\infty} \xi^{2}\left[\alpha_{3} \frac{d \tilde{F}^{0}}{\partial z}+2 \alpha_{2} \beta \tilde{F}^{0}\right] J_{1}(r \xi) d \xi \\
\hat{u}_{z}^{z}(r, z ; \beta)= & \int_{0}^{\infty} \xi\left[\alpha_{2} \frac{d}{d z}\left(2 \beta+\frac{d}{d z}\right)-\xi^{2}\left(1+\alpha_{1}\right)\right] \\
& \tilde{F}^{0} J_{0}(r \xi) d \xi
\end{aligned}
$$

and:

$$
\begin{aligned}
& \hat{\sigma}_{z r}^{z}(r, z ; \beta)= e^{2 \beta z} \int_{0}^{\infty} \xi^{2}\left[C _ { 4 4 } \left(\left(\alpha_{3}-\alpha_{2}\right) \frac{d^{2}}{d z^{2}}\right.\right. \\
&\left.\left.+\xi^{2}\left(1+\alpha_{1}\right)\right) \tilde{F}^{0}\right] J_{1}(r \xi) d \xi \\
& \hat{\sigma}_{z z}^{z}(r, z ; \beta)= e^{2 \beta z} \int_{0}^{\infty} \xi\left[C _ { 3 3 } \frac { d } { d z } \left(\alpha_{2} \frac{d}{\partial z}\left(2 \beta+\frac{d}{d z}\right)\right.\right. \\
&\left.-\xi^{2}\left(1+\alpha_{1}\right)+\alpha_{3} \frac{C_{13}}{C_{33}} \xi^{2}\right) \\
&\left.+2 \alpha_{2} \beta C_{13} \xi^{2}\right] \tilde{F}^{0} J_{0}(r \xi) d \xi \\
& \hat{\sigma}_{r r}^{z}(r, z ; \beta)+ \frac{2 e^{2 \beta z} C_{66}}{r} \hat{u}_{r}^{z}=e^{2 \beta z} \int_{0}^{\infty} \xi\left[\frac { d } { d z } \left(C_{11} \alpha_{3} \xi^{2}\right.\right. \\
&\left.\left.+\alpha_{2} C_{13} \frac{d}{d z}\left(2 \beta+\frac{d}{d z}\right)\right)+2 \alpha_{2} \beta C_{12} \xi^{2}\right] \\
& \tilde{F}^{0} J_{0}(r \xi) d \xi, \\
&-\left.C_{13} \xi^{2}\left(1+\alpha_{1}\right)+\alpha_{2} C_{13} \frac{d}{d z}\left(2 \beta+\frac{d}{d z}\right)\right) \\
& \hat{\sigma}_{\theta \theta}^{z}(r, z ; \beta)- 2 e^{2 \beta z} C_{66} \hat{u}_{r}^{z}=e^{2 \beta z} \\
& r C_{12} \alpha_{3} \xi^{2}-C_{13} \xi^{2}\left(1+\alpha_{1}\right) \\
&\left.\xi^{2}\right] \tilde{F}^{0} J_{0}(r \xi) d \xi
\end{aligned}
$$

where symbols $\hat{u}_{i}^{z}$ and $\hat{\sigma}_{i k}^{z}(i, k=r, z)$ indicate the displacement and stress Green's functions, respectively, and superscript $z$ indicates the direction of applied point-load. 


\subsection{Patch-load}

For a vertical circular patch-load of radius $a$ and resultant $F_{v}$, displacement and stress Green's functions may be obtained by Eqs. (33) and (34) with the following relation for loading function:

$$
R(r)=\frac{F_{v}}{\pi a^{2}} \rightarrow R^{0}(\xi)=F_{v} \frac{J_{1}(a \xi)}{\pi \xi a} .
$$

\subsection{Ring-load}

Similar to the patch-load analysis, the displacement and stress responses under the action of a vertical ringload acting at radius $a$ and resultant $F_{v}$ are given by Eqs. (33) and (34) with the following adaptation for loading function:

$$
R(r)=F_{v} \delta(r-a) \rightarrow R^{0}(\xi)=\frac{F_{v} J_{0}(a \xi)}{2 \pi} .
$$

\section{Special cases}

By means of the previously obtained solution for an exponentially graded transversely isotropic tri-material, some special cases of interest may be degenerated as follows.

\subsection{Exponentially graded transversely isotropic bi-material}

To obtain the formulation of an exponentially graded transversely isotropic bi-material, one may set $h=$ 0 , which means omitting the middle finite layer or substituting $C_{i j}^{I}=C_{i j}^{I I}$ and $\beta^{I}=\beta^{I I}$. In this case, by setting $\beta^{I}=\beta^{I I}=0$, the static Green's functions of a homogeneous transversely isotropic bi-material are obtained as in [28].

\subsection{Homogeneous transversely isotropic tri-materials}

Upon setting $\beta^{I}=\beta^{I I}=\beta^{I I I}=0$, the solutions for homogeneous transversely isotropic tri-materials are obtained as in [23] in static condition.

\subsection{Exponentially graded half-space} Considering $C_{i j}^{I} \rightarrow 0, \beta^{I I}=\beta^{I I I}=\beta$, and $C_{i j}^{I I}=$ $C_{i j}^{I I I}=C_{i j}$, under the action of surface or buried loads, Green's functions of an exponentially graded transversely isotropic half-space [9] or incompressible isotropic solid [13] are obtained.

\subsection{Homogeneous finite layer on a rigid half-space}

By applying load at $z=0$ and setting $C_{i j}^{I} \rightarrow 0$ and $C_{i j}^{I I I} \rightarrow \infty$, the response of a functionally graded finite layer on a rigid base is obtained which is exactly identical to the results in [24] and [27] for a finite layer on a rough-rigid half-space.

\section{Numerical evaluation}

In the previous section, the axisymmetric Green's functions associated with different load configurations have been expressed in terms of one-dimensional semiinfinite integrals. Since the closed-form integration is not possible [29-33], a numerical quadrature technique has to be adopted. For such evaluations, it is essential to consider the oscillatory nature of the integration due to the presence of Bessel functions and the singular nature of stress transfer. Here, an adaptive quadrature rule has been utilized successfully by using Wolfram Mathematica software. For verification of the present study with the existing numerical solutions in the literature, three comparisons are made. First, for a homogeneous tri-material, vertical displacement of the medium due to point-load is determined and compared with the results given by Khojasteh et al. [23]. As shown in Figure 3, the solutions are identical. Also, under applied buried vertical patchload, the axial displacement along the depth of an exponentially graded half-space for different inhomogeneity parameters $(\beta)$ is compared with the results by Eskandari and Shodja [9] (Figure 4). Here, it is noteworthy that for an isotropic material, the elasticity constants may be reduced to $C_{11}=C_{33}=\lambda+2 \mu$, $C_{12}=C_{13}=\lambda$, and $C_{44}=C_{66}=\mu$ where $\lambda$ and $\mu$ are the Lame's constants of the isotropic solid. For an incompressible isotropic solid with the initial shear modulus of $\mu_{0}=3.33$, under applied patchload, vertical displacement along $z$-axis is shown in Figure 5 and is compared with the results obtained by Selavadurai and Katebi [13], which shows the good agreement of results.

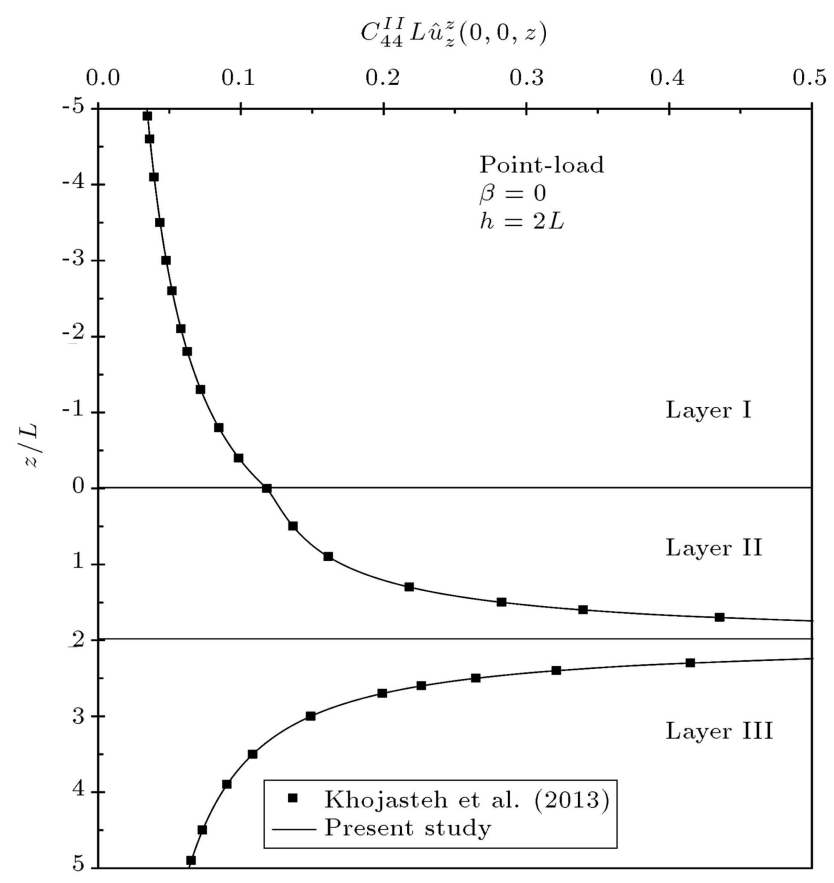

Figure 3. Displacement Green's function $\hat{u}_{z}^{z}$ along $z$-direction due to point-load compared with the result by Khojasteh et al. [23] for homogenous materials $(\beta L=0)$. 


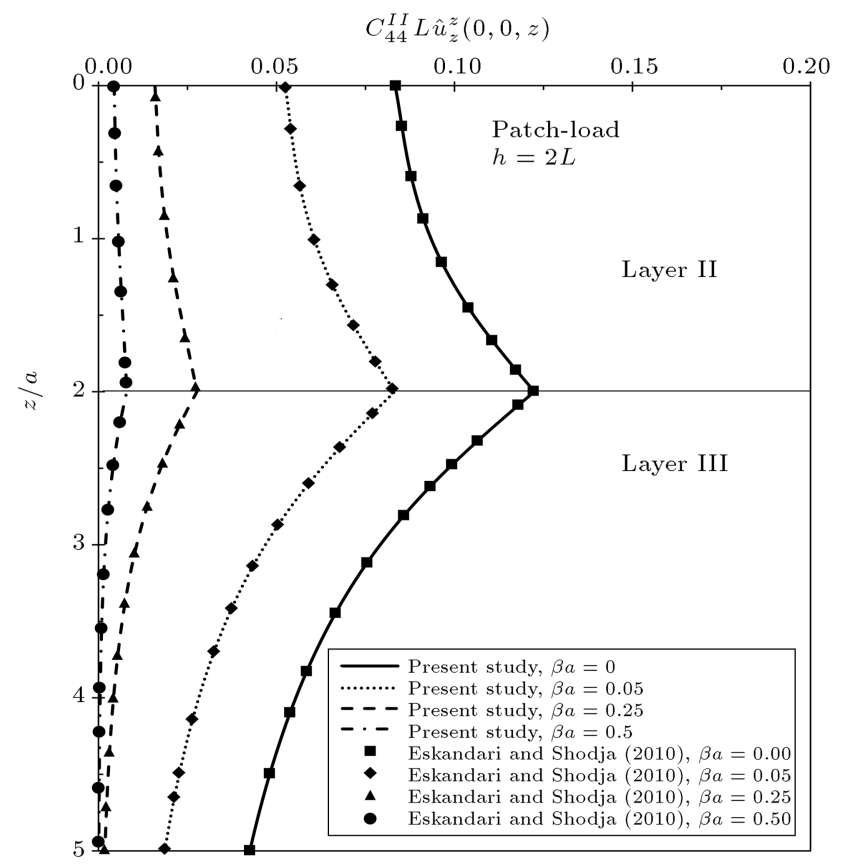

Figure 4. Displacement Green's function $\hat{u}_{z}^{z}$ along $z$-axis compared with the study by Eskandari and Shodja [9] for functionally graded transversely isotropic half space.

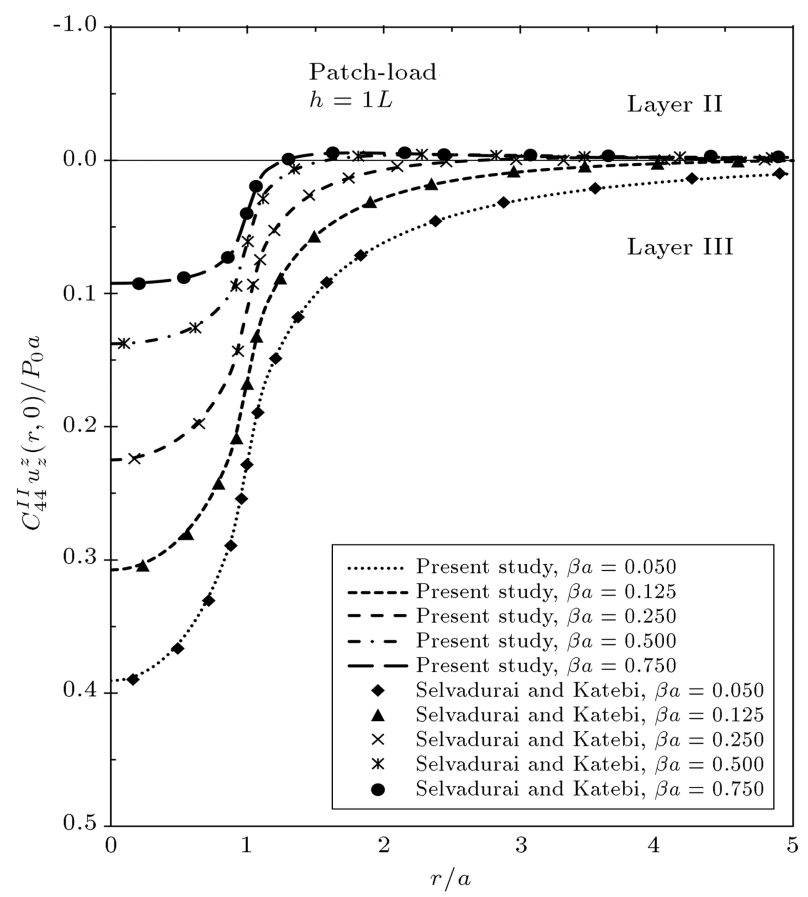

Figure 5. Displacement Green's function $\hat{u}_{z}^{z}$ along $r$-axis compared with the results by Selvadurai and Katebi [13] for incompressible isotropic half-space.

In this study, three different synthetic transversely isotropic materials are considered for constructing the parametric study of analysis results as listed in Table 1 .

In the following, three different material configurations in layers are considered:
Table 1. Elastic constants of transversely isotropic materials.

\begin{tabular}{cccccc}
\hline $\begin{array}{c}\text { Material } \\
\text { no. }\end{array}$ & $\begin{array}{c}\boldsymbol{C}_{\mathbf{1 1}} \\
(\mathbf{G} \mathbf{p a})\end{array}$ & $\begin{array}{c}\boldsymbol{C}_{\mathbf{1 2}} \\
(\mathbf{G} \mathbf{p a})\end{array}$ & $\begin{array}{c}\boldsymbol{C}_{\mathbf{1 3}} \\
(\mathbf{G} \mathbf{p a})\end{array}$ & $\begin{array}{c}\boldsymbol{C}_{\mathbf{3 3}} \\
(\mathbf{G} \mathbf{p a})\end{array}$ & $\begin{array}{c}\boldsymbol{C}_{\mathbf{4 4}} \\
(\mathbf{G} \mathbf{p a})\end{array}$ \\
\hline Material 1 & 5.6 & 1.6 & 1.8 & 10.9 & 2 \\
Material 2 & 5.5 & 1.5 & 1.8 & 15.9 & 2 \\
Material 3 & 5.4 & 1.4 & 1.7 & 25.9 & 2 \\
\hline
\end{tabular}

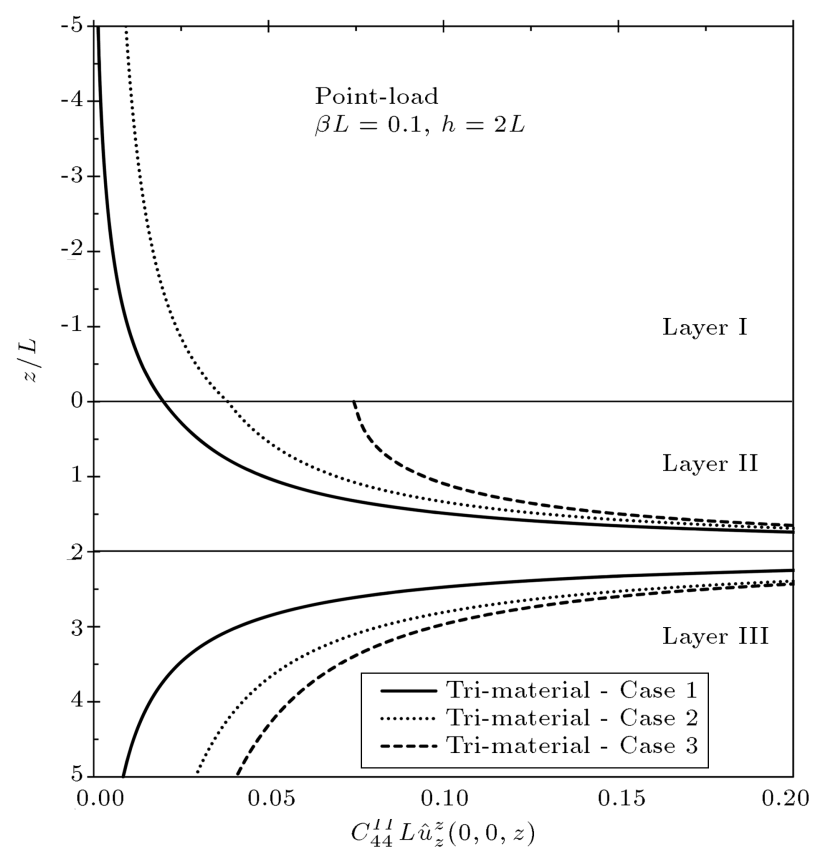

Figure 6. Displacement Green's function $\hat{u}_{z}^{z}$ along $z$-axis due to unit point-load $(\beta L=0.1)$.

- Case 1: Material 2 in all three layers;

- Case 2: Materials 1, 2, and 3 in Layers I, II, and III, respectively;

- Case 3: Similar to Case 2, Layer 1 is omitted, $C_{i j}^{I} \rightarrow$ 0 , which resembles the two-layered half-space or may be used for buried-load configuration analysis.

By considering the described cases, Figures 6 and 7 depict displacement Green's function $\hat{u}_{z}^{z}$ along the depth of point-load with $\beta * L=0.1$, thickness of middle layer $h=2 L$, and patch-load with the resultant of $1 / \pi, \beta * a=0.1$ and $h=2 a$, respectively, where $L$ is the unit of length. Based on the figures, one should notice the continuity at interface as well as the singularity of the response at point load location accompanying the significant effect of inhomogeneity parameter.

Figures 8 and 9 depict the influence of thickness of middle layer, $h$, on the displacement Green's function $\hat{u}_{z}^{z}$ along the depth and radial directions, respectively. For higher thickness of middle layer, one may observe the lower displacement response which is mainly the 


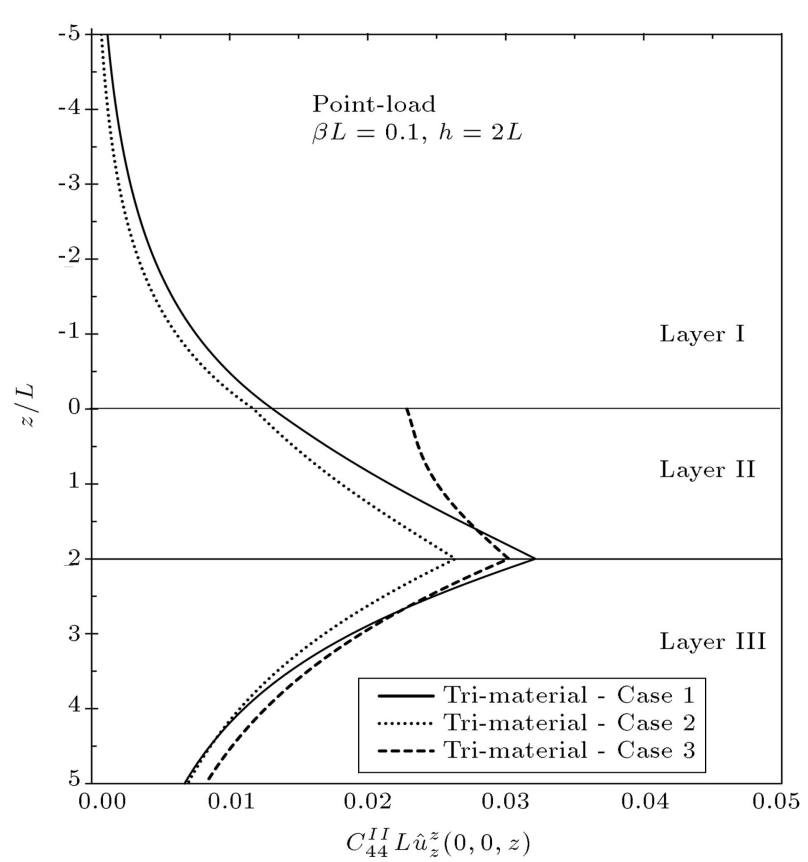

Figure 7. Displacement Green's function $\hat{u}_{z}^{z}$ along $z$-axis due to patch-load with resultant $1 / \pi(\beta a=0.1)$.

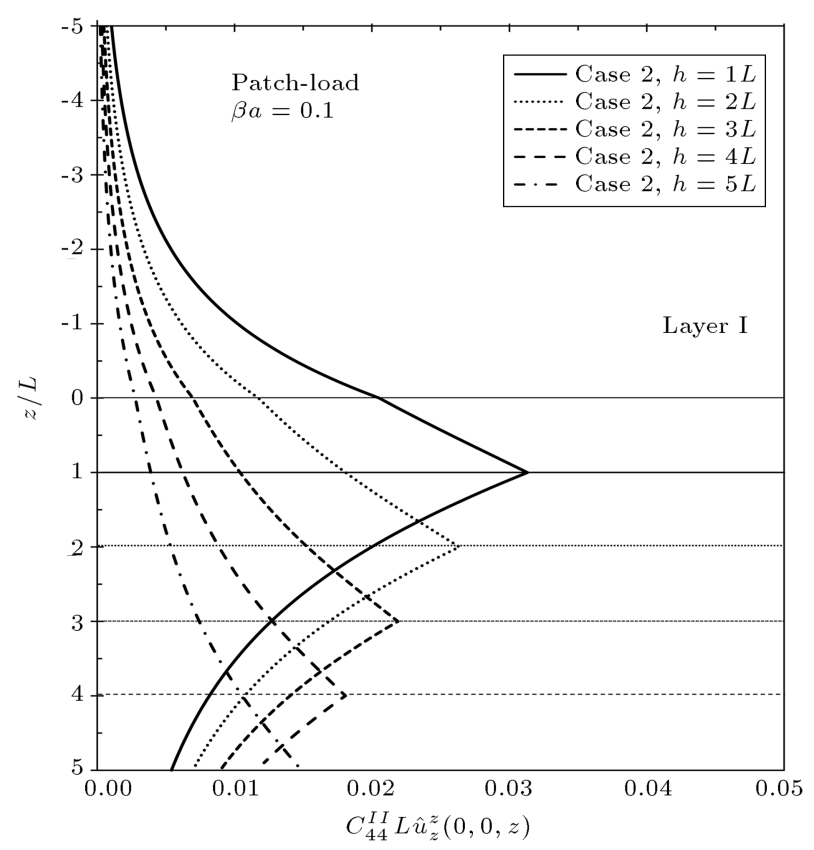

Figure 8. Displacement Green's function $\hat{u}_{z}^{z}$ along $z$-direction due to point-load for different middle layer thicknesses $(\beta a=0.1)$.

result of exponential increase of elasticity constant of the layer.

To survey the effect of inhomogeneity parameter only on the middle layer in Case 1, the inhomogeneity parameter has been changed in Figures 10 to 12, with $\beta=0$ representing the homogeneous layer. Figure 10 depicts vertical displacement $\hat{u}_{z}^{z}$ of the medium under applied point-load along depth, which shows the lower

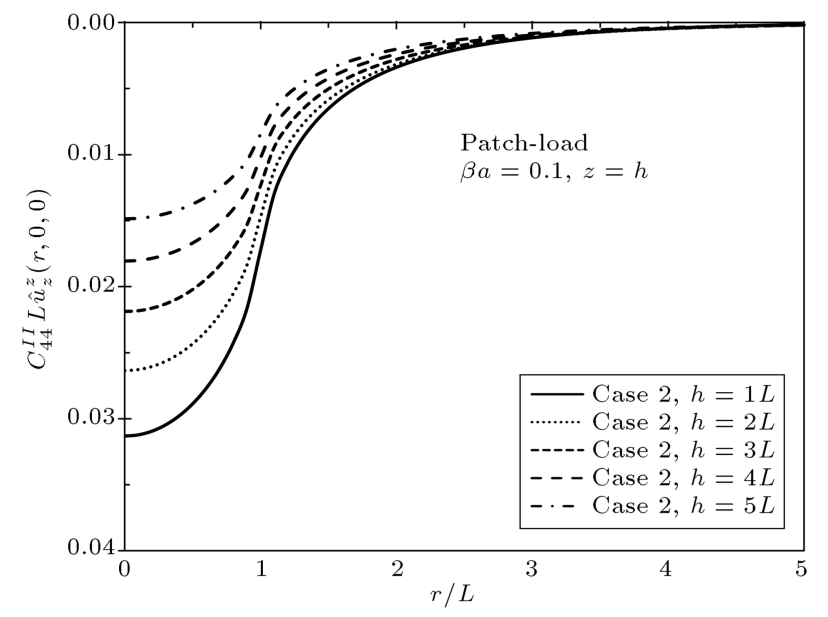

Figure 9. Displacement Green's function $\hat{u}_{z}^{z}$ along $r$-direction due to patch-load for different middle layer thicknesses $(\beta a=0.1)$.

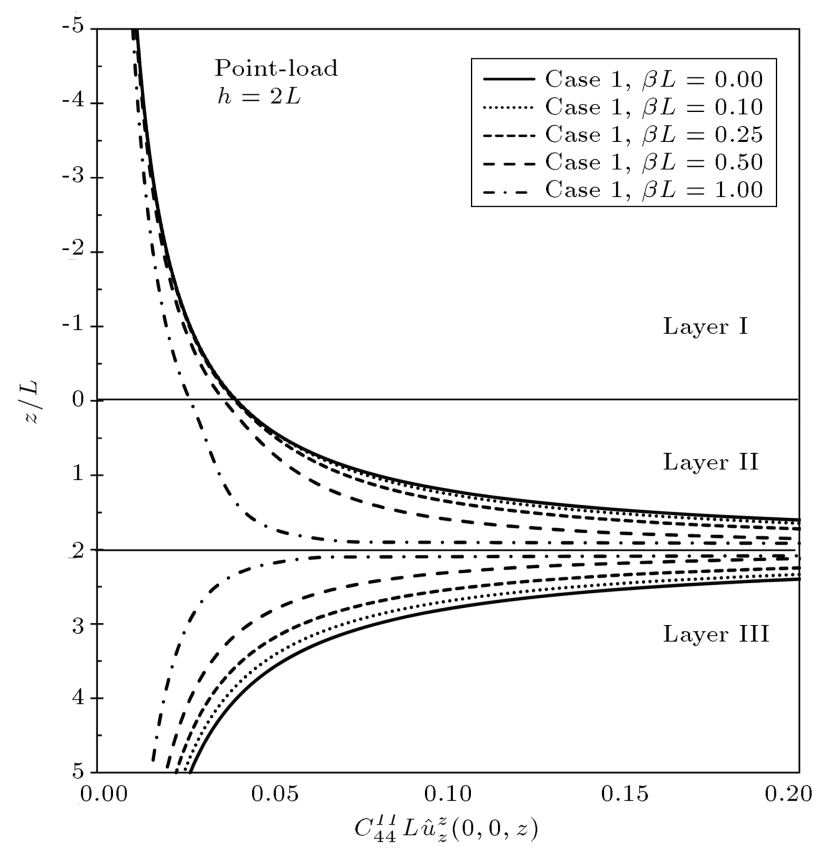

Figure 10. Displacement Green's function $\hat{u}_{z}^{z}$ along $z$-axis due to unit point-load.

displacement in all three layers of higher inhomogeneity parameter $\beta$ as a result of higher stiffness of the middle layer. A similar trend is observed for the response in Figure 11 for displacement under patch-load. For the sustained vertical stress in the medium, Figure 12 is presented which shows a more rapid stress transfer by the increase of $\beta$.

For studying the response of an inhomogeneous finite layer on a rigid base, Figures 13-17 are included. In these figures, Layer $\mathrm{I}$ is omitted (i.e., $C_{i j}^{I} \rightarrow 0$ ), Material 1 is used in Layer II, and the material parameters are highly increased for Layer III to resemble the rigid base layer (i.e., $C_{i j}^{I I I} \rightarrow \infty$ ). Also, the boundary conditions on the interface of the two layers are defined 


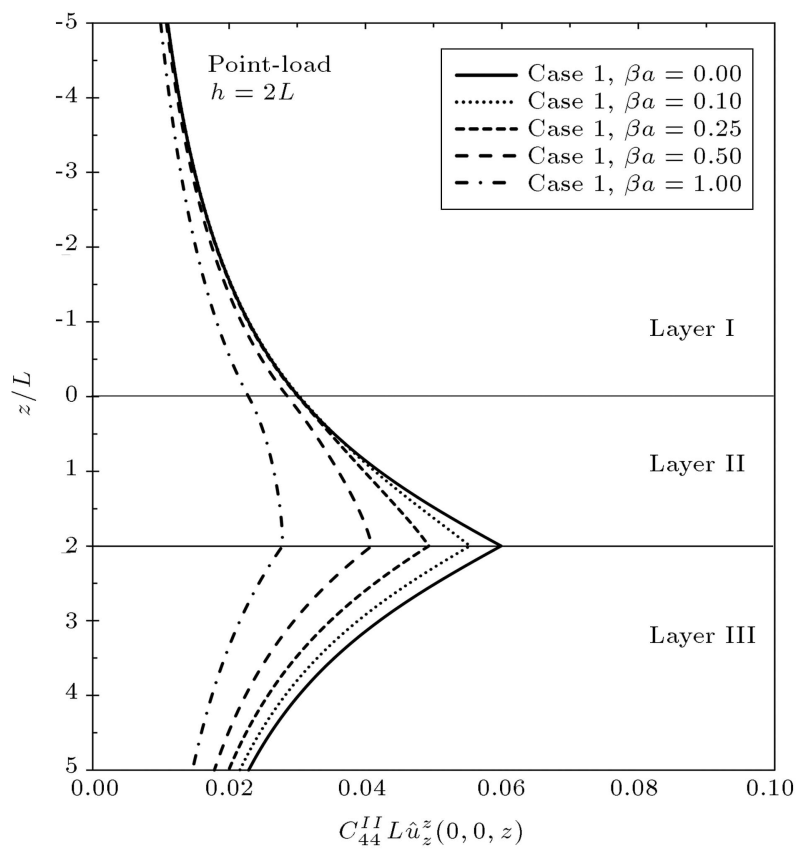

Figure 11. Displacement Green's function $\hat{u}_{z}^{z}$ along $z$-axis due to patch-load with resultant $1 / \pi$.

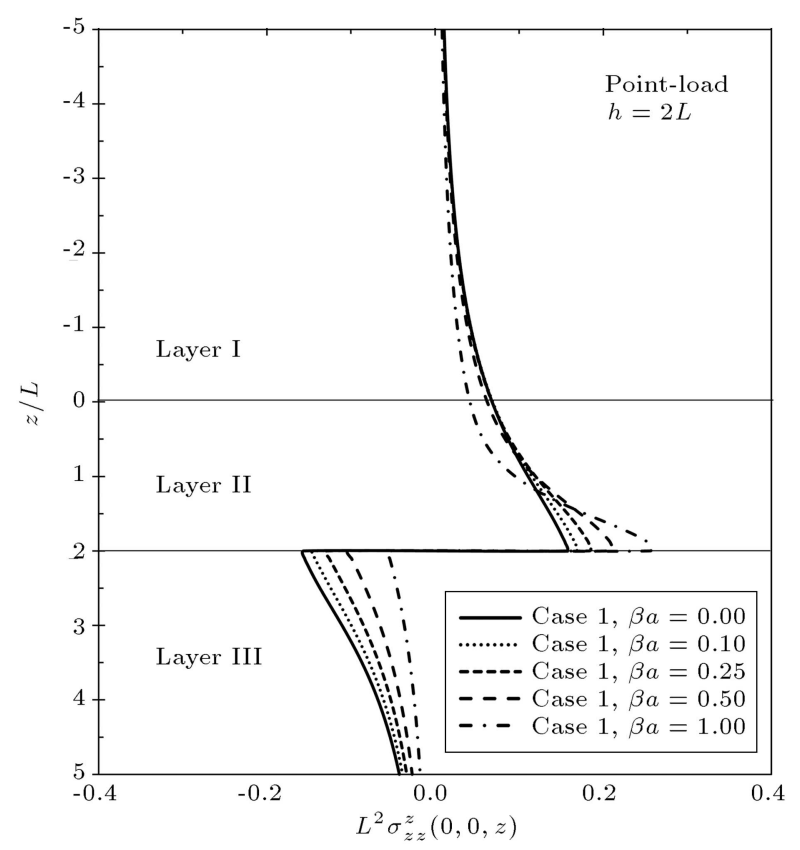

Figure 12. Stress Green's function $\hat{\sigma}_{z z}^{z}$ along $z$-axis due to patch-load with resultant $1 / \pi$.

as rough and smooth, which means an interface with infinite and without friction capacity, respectively.

Figure 13 depicts the vertical stress distribution due to patch-load in a transversely isotropic finite layer bonded to a rigid base, compared with the solutions by Small and Booker [24]. Figures 14 and 15 illustrate the influence of inhomogeneity parameter on vertical stress distribution $\left(\hat{\sigma}_{z z}^{z}\right)$ due to a patch-load in vertical direction and rough/smooth-rigid conditions, respec-

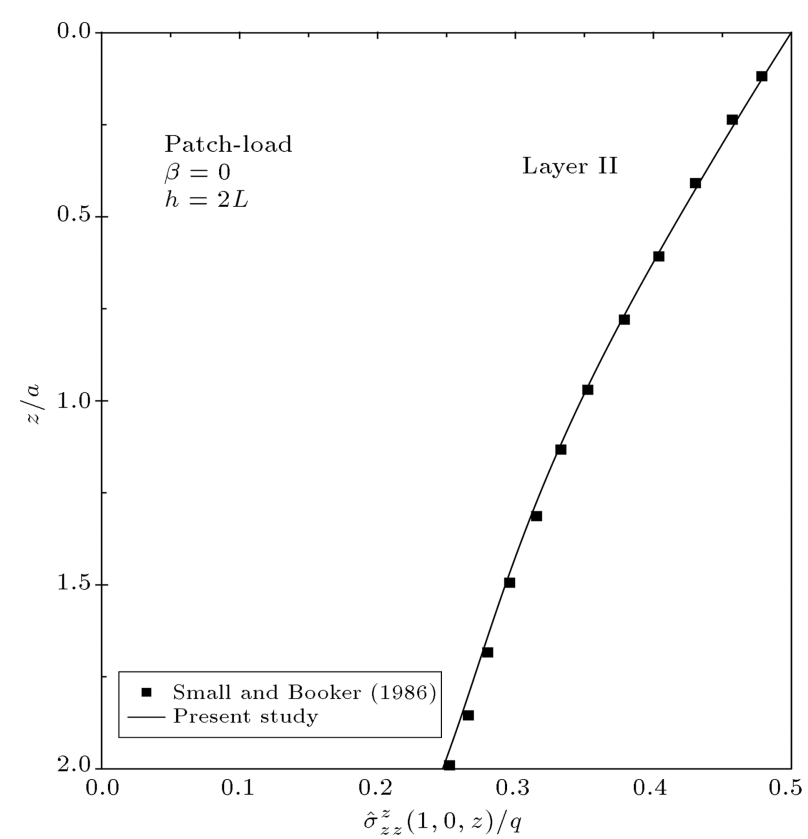

Figure 13. Stress Green's function $\hat{\sigma}_{z z}^{z}$ beneath the edge of a circular loading along $z$-direction for finite layer on a rigid half-space with rough-rigid conditions compared with the results by Small and Booker [24].

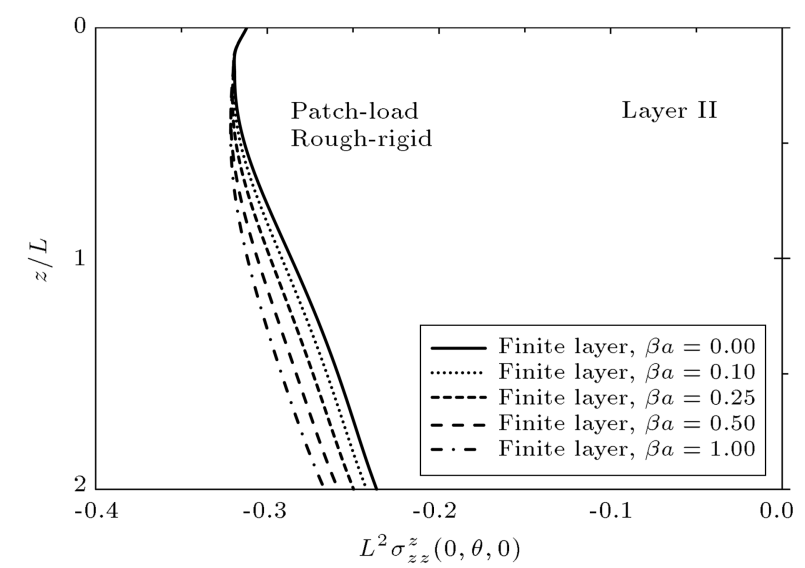

Figure 14. Stress Green's function $\hat{\sigma}_{z z}^{z}$ along $z$-axis due to patch-load with resultant $1 / \pi$ for a finite layer on a rigid half-space with rough-rigid condition.

tively. Based on the figures, one should notice the significant effect of inhomogeneity as in stiffer medium, i.e. the higher inhomogeneity factor is, the higher stress reaches the interface. The influence of inhomogeneity on vertical displacement of solid $\left(\hat{u}_{z}^{z}\right)$ is shown in Figure 16. From the figure, one should notice the decrease of displacement along the depth and effect of rough condition on the decrease of displacement, especially for lower $\beta$. Finally, Figure 17 represents the radial displacement component $\left(\hat{u}_{r}^{z}\right)$ along the radial direction at the interface with the smooth-rigid condition. From the figure, one should notice the significant reduction of displacement as the effect of higher stiffness of the 


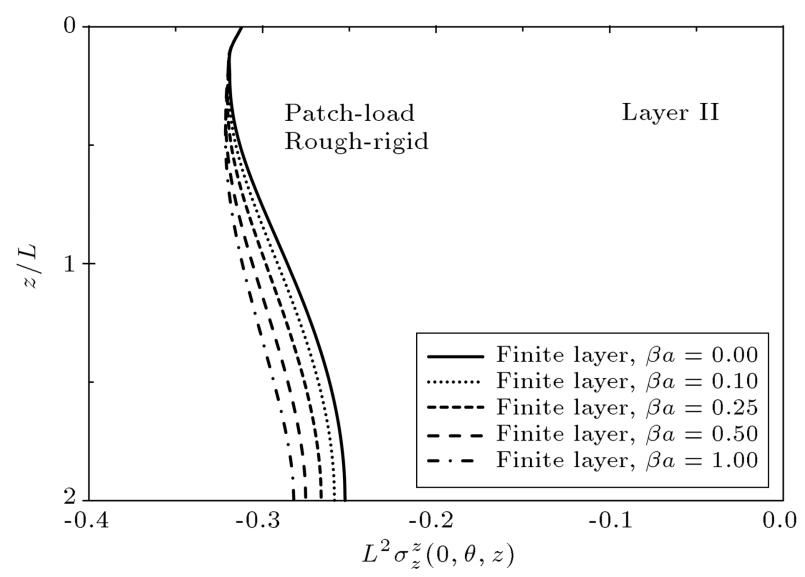

Figure 15. Stress Green's function $\hat{\sigma}_{z z}^{z}$ along $z$-axis due to patch-load with resultant $1 / \pi$ for a finite layer on a rigid half-space with smooth-rigid condition.

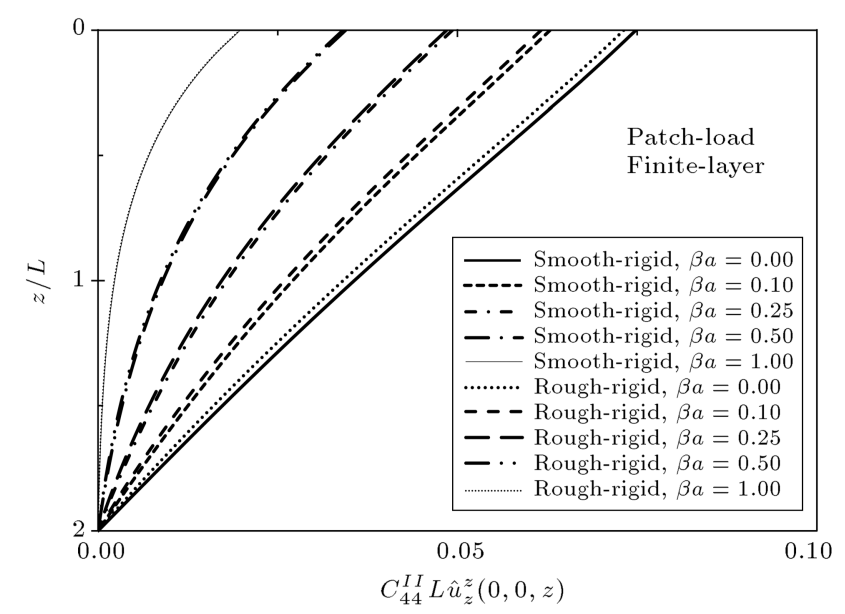

Figure 16. Displacement Green's function $\hat{u}_{z}^{z}$ along $z$-axis due to patch-load with resultant $1 / \pi$ for a finite layer with rough-rigid and smooth-rigid conditions.

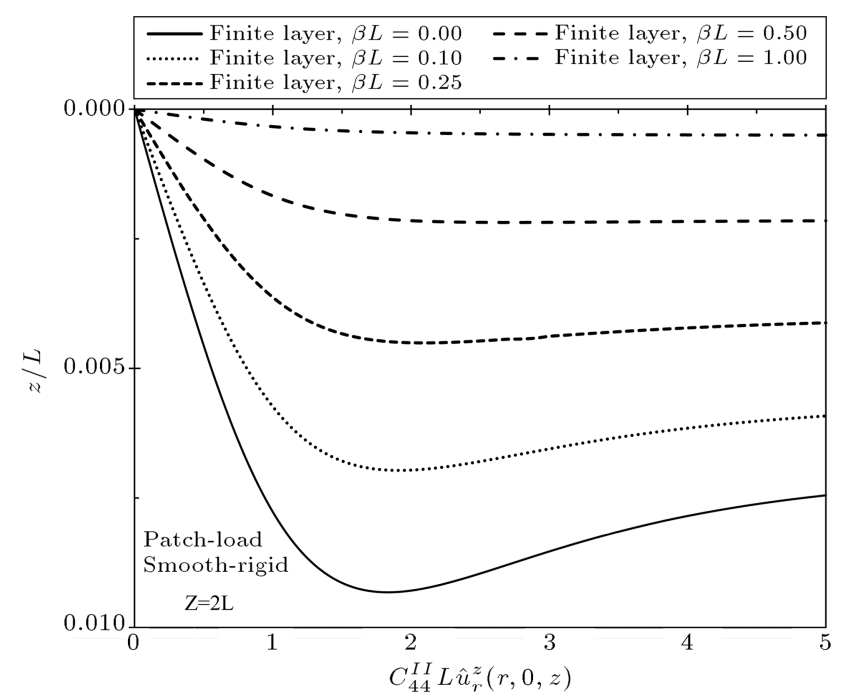

Figure 17. Displacement Green's function $\hat{u}_{r}^{z}$ along $r$-axis direction due to the patch-load and for a finite layer with smooth-rigid conditions. layer. It is trivial that $\hat{u}_{r}^{z}$ at interface tends to zero for the case of rough-rigid condition.

\section{Conclusion}

The axisymmetric response of an exponentially graded transversely isotropic tri-material elastic solid due to point-load and patch-load in static condition is presented in this study. By use of one displacement potential function and Hankel transform, different components of response of the medium under applied loads are given in terms of infinite line-integrals. These equations are useful for effective boundary element formulations of elastostatic problems and mixed boundary value problem formulations in inhomogeneous and anisotropic composites. These Green's functions can be degenerated analytically and numerically for different cases of exponentially graded transversely isotropic bimaterial, homogeneous full-space tri-material, exponentially graded half space, and special case of finite layer on a rigid half-space. Numerical examples show the significant influence of the thickness of middle layer and the degree of inhomogeneity of the material on all aspects of the response.

\section{Nomenclature}

\begin{tabular}{|c|c|}
\hline$a$ & Loading radius \\
\hline$A_{m}, \ldots, D_{m}$ & Constants of integration \\
\hline$C_{i j}$ & Elasticity constants \\
\hline$F$ & Potential function \\
\hline FGM & Functionally Graded Material \\
\hline$F_{v}$ & Vertical point-load resultant value \\
\hline$h$ & Middle layer thickness \\
\hline$J_{m}$ & $\begin{array}{l}\text { Bessel function of the first kind and } \\
m \text {-th order }\end{array}$ \\
\hline$m$ & Hankel integral transform \\
\hline$r$ & Radial coordinate \\
\hline$R(r)$ & $\begin{array}{l}\text { Specified interfacial traction in } z \text {-axis } \\
\text { direction }\end{array}$ \\
\hline$s_{1}, s_{2}$ & Roots of strain energy function \\
\hline$u_{r}$ & $\begin{array}{l}\text { Displacement components in } r \text { - } \\
\text { directions }\end{array}$ \\
\hline$u_{z}$ & $\begin{array}{l}\text { Displacement components in } z \text { - } \\
\text { directions }\end{array}$ \\
\hline$z_{0}$ & Transformed loading coefficient \\
\hline$\beta$ & $\begin{array}{l}\text { Exponential factor characterizing the } \\
\text { degree of material properties gradient }\end{array}$ \\
\hline$\Pi_{0}$ & Arbitrary area \\
\hline$\Pi_{h}$ & Arbitrary area \\
\hline$\sigma_{i j}$ & Stress components \\
\hline$\lambda$ & Lambs constant \\
\hline$\mu$ & Lambs constant \\
\hline
\end{tabular}




\section{References}

1. Birman, V. and Byrd, L.W. "Modeling and analysis of functionally graded materials and structures" , $A M R$. ASME., 60(50), pp. 195-216 (2007).

2. Wang, C.D., Tzeng, C.S., Pan, E. and Liao, J.J. "Displacements and stresses due to a vertical point load in an inhomogeneous transversely isotropic halfspace", Int. J. Rock Mech. Min., 40(5), pp. 667-685 (2003).

3. Wang, C.D., Pan, E., Tzeng, C.S., Han, F. and Liao, J.J. "Displacements and stresses due to a uniform vertical circular load in an inhomogeneous crossanisotropic half-space" , Int. J. of Geomechanics., 6(1), p. 110 (2006).

4. Wang, C.D. and Tzeng, C.S. "Displacements and stresses due to non-uniform circular loadings in an inhomogeneous cross-anisotropic material", Mech. Res. Commun., 36, pp. 921-932 (2009).

5. Martin, P.A., Richardson, J.D., Gray, L.J. and Berger, J.R. "On Green's function for a three-dimensional exponentially graded elastic solid", Pro. R. Soc. London, Ser. A., 458, pp. 1931-1947 (2002).

6. Pan, E. and Yang, B. "Three-dimensional interfacial Green's functions in anisotropic bi-materials" , Appl. Math. Model., 27, pp. 307-326 (2003).

7. Chan, Y.S., Grey, L.J., Kaplan, T. and Paulino, G.H. "Green's function for a two-dimensional exponentially graded elastic medium" , Pro. R. Soc. London, Ser. A., pp. 1689-1706 (2004).

8. Sallah, O.M., Gray, L.J., Amer, M.A. and Matbuly, M.S. "Green's function expansion for exponentially graded elasticity" , Int. J. Numer. Meth. Eng., 82, pp. 756-722 (2010)

9. Eskandari, M. and Shodja, H.M. "Green's functions of an exponentially graded transversely isotropic halfspace" , Int. J. of Solids Struct., 47, pp. 1537-1545 (2010).

10. Kashtalyan, M. and Rushchitsky, J.J. "Revisiting displacement functions in three-dimensional elasticity of inhomogeneous media" , Int. J. of Solids Struct., 46, pp. 3463-3470 (2009).

11. Wang, C.D., Lin, Y.T., Jeng, Y.S. and Ruan Z.W. "Wave propagation in an inhomogeneous crossanisotropic medium", Int. J. for NAMG., 34, p. n/an/a (2009).

12. Eskandari-Ghadi, M. and Amiri-Hezaveh, A. "Wave propagations in exponentially graded transversely isotropic half-space with potential function method", Mechanics of Materials., 68, pp. 275-292 (2014).

13. Selvadorai, A.P.S. and Katebi, A. "Mindlin's problem for an incompressible elastic half-space with an exponential variation in the linear elastic shear modulus", Int. J. ES., 65, pp. 9-21 (2013).

14. Katebi, A. and Selvadurai, A.P.S. "A frictionless contact problem for a flexible circular plate and an incompressible non-homogeneous elastic halfspace", Int. J of Mech. Sci., 90, pp. 239-245 (2015).
15. Selvadurai, A.P.S. and Katebi, A. "An adhesive contact problem for an incompressible non-homogeneous elastic halfspace", Int. J of Acta Mechanica, 226(2), pp. 249-265 (2015).

16. Ke, L.-L. and Wang, Y.-S. "Two-dimensional sliding frictional contact of functionally graded materials", European J. of Mechanics - A/Solids., 26(1), pp. 171188 (2007).

17. Liu, T., Wang, Y. and Xing, Y. "Fretting contact of two elastic solids with graded coatings under torsion", Int. J. of Solids Struct., 49(10), pp. 1283-1293 (2012).

18. Chen, P. and Chen, S. "Partial slip contact between a rigid punch with an arbitrary tip-shape and an elastic graded solid with a finite thickness", Int. J. of Mechanics of Materials, 59, pp. 24-35 (2013).

19. Volkov, S., Aizikovich, S., Wang, Y.S. and Fedotov, I. "Analytical solution of axisymmetric contact problem about indentation of a circular indenter into a soft functionally graded elastic layer" , Int. J. of Acta. Mechanica, 29(2), pp. 196-201 (2013).

20. Liu, T.-J., Xing, Y.-M. and Wang, Y.-S. "The axisymmetric contact problem of a coating/substrate system with a graded interfacial layer under a rigid spherical punch" , Int. J. of Mathematics and Mechanics of Solids, 21(3), pp. 383-399 (2014).

21. Matysiak, S.J., Kulchytsky-Zhyhailo, R. and Perkowski, D.M. "Reissner-Sagoci problem for a homogeneous coating on a functionally graded halfspace", Int. J. of Mechanics Research Communications, 38(4), pp. 320-325 (2011).

22. Kulchytsky-Zhyhailo, R. and Bajkowski, A.S. "Stresses in coating with gradient interlayer caused by contact loading", Int. J. of Acta Mechanica et Automatica, 8(1), pp. 33-37 (2014).

23. Khojasteh, A., Rahimian, M. and Eskandari, M. "Three-dimensional dynamic Green's functions in transversely isotropic tri-materials", Appl. Math. Model., 37, pp. 3164-3180 (2013).

24. Small, J.C. and Booker, J.R. "Finite layer analysis of layered elastic materials using a flexibility approach part 2-circlular and rectangular loading", Int. $J$. Numer. Meth. Eng., 23, pp. 959-978 (1986).

25. Lekhnitskii, S.G., Theory of Elasticity of an Anisotropic Elastic Body, Holden Day., San Francisco (1964).

26. Sneddon, I.N. The Use of Integral Transforms, McGraw-Hill., New York (1972).

27. Pan, E. "Static response of a transversely isotropic and layered half-space to general surface loads" , Phys. Earth Planet Inter., 54, pp. 353-363 (1988).

28. Khojasteh, A. and Rahimian, M. "Three-dimensional dynamic Green's functionsin transversely isotropic bimaterials" , Int. J. of Solids Struct., 45, pp. 4952-4972 (2008).

29. Apsel, R.J. and Luco, J.E. "On the Green's functions for a layered half space. Part II", BSSA., 73(4), pp. $931-820$ (1983). 
30. Pak, R.Y.S. and Guzina, B.B. "Three-dimensional Green's functions for a multi-layered halfspace displacement potentials", J. Eng. Mech., 128(4), pp. 449461 (2002).

31. Rajapakse, R.K.N.D. and Wang, Y. "Green's functions for transversely isotropic elastic halfspace" , J. Eng. Mech. ASCE., 119(9), pp. 1724-1746 (1993).

32. Rahimian, M., Eskandari-Ghadi, M., Pak, R.Y.S. and Khojasteh, A. "Elastodynamic potential method for transversely isotropic solid", J. Eng. Mech. ASCE., 133(10), pp. 1134-1145 (2007).

33. Khojasteh, A., Rahimian, M., Eskandari, M. and Pak, R.Y.S. "Three-dimensional dynamic Green's functions for a multilayered transversely isotropic half-space", Int. J. of Solids Struct., 48, pp. 801-820 (2011).

\section{Appendix A}

For the case of general tri-material solid analysis, matrix $I(\xi)$ is written as Eq. (A.1) as shown in Box A.1. For Rough-Rigid interface formulation in finite layer analysis it is written as:

$$
I(\xi)=\left[\begin{array}{cccc}
\vartheta_{1}^{I I} & \omega_{1}^{I I} & \vartheta_{2}^{I I} & \omega_{2}^{I I} \\
\nu_{1}^{I I} & v_{1}^{I I} & \nu_{2}^{I I} & v_{2}^{I I} \\
\psi_{1}^{I I} & \Omega_{1}^{I I} & \psi_{2}^{I I} & \Omega_{2}^{I I} \\
\Phi_{1}^{I I} & \Theta_{1}^{I I} & \Phi_{2}^{I I} & \Theta_{2}^{I I}
\end{array}\right],
$$

for Smooth-Rigid analysis of finite layer, it may be written as:

$$
I(\xi)=\left[\begin{array}{cccc}
\vartheta_{1}^{I I} & \omega_{1}^{I I} & \vartheta_{2}^{I I} & \omega_{2}^{I I} \\
\nu_{1}^{I I} & v_{1}^{I I} & \nu_{2}^{I I} & v_{2}^{I I} \\
\psi_{1}^{I I} & \Omega_{1}^{I I} & \psi_{2}^{I I} & \Omega_{2}^{I I} \\
p_{1}^{I I} & q_{1}^{I I} & p_{2}^{I I} & q_{2}^{I I}
\end{array}\right] .
$$

The terms $\varphi_{i}^{I}, \ldots, t_{i}^{I I I}$ are defined as follows:

$$
\begin{aligned}
& \varphi_{i}^{I}=2 \alpha_{2}^{I} \beta^{I}+\alpha_{3}^{I}\left(\beta^{I}+\lambda_{i}^{I}\right), \\
& \varphi_{i}^{I I}=2 \alpha_{2}^{I I} \beta^{I I}-\alpha_{3}^{I I}\left(\beta^{I I}-\lambda_{i}^{I I}\right), \\
& \theta_{i}^{I I}=2 \alpha_{2}^{I I} \beta^{I I}-\alpha_{3}^{I I}\left(\beta^{I I}+\lambda_{i}^{I I}\right), \\
& \theta_{i}^{I I I}=2 \alpha_{2}^{I I I} \beta^{I I I}-\alpha_{3}^{I I I}\left(\beta^{I I I}+\lambda_{i}^{I I I}\right), \\
& \vartheta_{i}^{I}=2 \alpha_{2}^{I} \beta^{I}\left(\beta^{I}+\lambda_{i}^{I}\right)-\left(1+\alpha_{1}^{I}\right) \xi^{2}+\alpha_{2}^{I}\left(\beta^{I}+\lambda_{i}^{I}\right)^{2}, \\
& \vartheta_{i}^{I I}=-2 \alpha_{2}^{I I} \beta^{I I}\left(\beta^{I I}-\lambda_{i}^{I I}\right)-\left(1+\alpha_{1}^{I I}\right) \xi^{2} \\
& +\alpha_{2}^{I I}\left(\beta^{I I}-\lambda_{i}^{I I}\right)^{2} \\
& \vartheta_{i}^{I I I}=-2 \alpha_{2}^{I I I} \beta^{I I I}\left(\beta^{I I I}+\lambda_{i}^{I I I}\right)-\left(1+\alpha_{1}^{I I I}\right) \xi^{2} \\
& +\alpha_{2}^{I I I}\left(\beta^{I I I}+\lambda_{i}^{I I I}\right)^{2} \\
& \omega_{i}^{I I}=-2 \alpha_{2}^{I I} \beta^{I I}\left(\beta^{I I}+\lambda_{i}^{I I}\right)-\left(1+\alpha_{1}^{I I}\right) \xi^{2} \\
& +\alpha_{2}^{I I}\left(\beta^{I I}+\lambda_{i}^{I I}\right)^{2} \\
& \omega_{i}^{I I I}=-2 \alpha_{2}^{I I I} \beta^{I I I}\left(\beta^{I I I}+\lambda_{i}^{I I I}\right)-\left(1+\alpha_{1}^{I I I}\right) \xi^{2} \\
& +\alpha_{2}^{I I I}\left(\beta^{I I I}+\lambda_{i}^{I I I}\right)^{2} \\
& \eta_{i}^{I}=C_{44}^{I}\left[2 \alpha_{2}^{I} \beta^{I}\left(\beta^{I}+\lambda_{i}^{I}\right)+\alpha_{3}^{I}\left(\beta^{I}+\lambda_{i}^{I}\right)^{2}-\vartheta_{i}^{I}\right], \\
& \eta_{i}^{I I}=C_{44}^{I I}\left[-2 \alpha_{2}^{I I} \beta^{I I}\left(\beta^{I I}-\lambda_{i}^{I I}\right)\right. \\
& \left.+\alpha_{3}^{I I}\left(\beta^{I I}-\lambda_{i}^{I I}\right)^{2}-\vartheta_{i}^{I I}\right], \\
& \xi_{i}^{I I}=C_{44}^{I I}\left[-2 \alpha_{2}^{I I} \beta^{I I}\left(\beta^{I I}+\lambda_{i}^{I I}\right)\right. \\
& \left.+\alpha_{3}^{I I}\left(\beta^{I I}+\lambda_{i}^{I I}\right)^{2}-\omega_{i}^{I I}\right],
\end{aligned}
$$

$$
I(\xi)=\left[\begin{array}{cccccccc}
\varphi_{1}^{I} & \varphi_{2}^{I} & -\varphi_{1}^{I I} & -\theta_{1}^{I I} & -\varphi_{2}^{I I} & -\theta_{2}^{I I} & 0 & 0 \\
\vartheta_{1}^{I} & \vartheta_{2}^{I I} & -\vartheta_{1}^{I I} & -\omega_{1}^{I I} & -\vartheta_{2}^{I I} & -\omega_{2}^{I I} & 0 & 0 \\
0 & 0 & \Phi_{1}^{I I} & \Theta_{1}^{I I} & \Phi_{2}^{I I} & \Theta_{2}^{I I} & -\Theta_{1}^{I I I} & -\Theta_{2}^{I I I} \\
0 & 0 & \psi_{1}^{I I} & \Omega_{1}^{I I} & \psi_{2}^{I I} & \Omega_{2}^{I I} & -\Omega_{1}^{I I I} & -\Omega_{2}^{I I I} \\
\eta_{1}^{I} & \eta_{2}^{I I} & -\eta_{1}^{I I} & -\xi_{1}^{I I} & \eta_{2}^{I I} & -\xi_{2}^{I I} & 0 & 0 \\
\nu_{1}^{I} & \nu_{2}^{I I} & -\nu_{1}^{I I} & -v_{1}^{I I} & -\nu_{2}^{I I} & -v_{2}^{I I} & 0 & 0 \\
0 & 0 & p_{1}^{I I} & q_{1}^{I I} & p_{2}^{I I} & q_{2}^{I I} & -q_{1}^{I I I} & -q_{2}^{I I I} \\
0 & 0 & n_{1}^{I I} & t_{1}^{I I} & n_{2}^{I I} & t_{2}^{I I} & -t_{1}^{I I I} & -t_{2}^{I I I}
\end{array}\right]
$$




$$
\begin{aligned}
& \xi_{i}^{I I I}=C_{44}^{I I I}\left[-2 \alpha_{2}^{I I I} \beta^{I I I}\left(\beta^{I I I}+\lambda_{i}^{I I I}\right)\right. \\
& \left.+\alpha_{3}^{I I I}\left(\beta^{I I I}+\lambda_{i}^{I I I}\right)^{2}-\omega_{i}^{I I I}\right], \\
& v_{i}^{I}=\left(B^{I}+\lambda_{i}^{I}\right) C_{33}^{I} \vartheta_{i}^{I}+C_{13}^{I} \xi^{2} \varphi_{i}^{I}, \\
& v_{i}^{I I}=-\left(B^{I I}-\lambda_{i}^{I I}\right) C_{33}^{I I} \vartheta_{i}^{I I}+C_{13}^{I I} \xi^{2} \varphi_{i}^{I I}, \\
& \nu_{i}^{I I}=-\left(B^{I I}+\lambda_{i}^{I I}\right) C_{33}^{I I} \omega_{i}^{I I}+C_{13}^{I I} \xi^{2} \theta_{i}^{I I}, \\
& \nu_{i}^{I I I}=-\left(B^{I I I}+\lambda_{i}^{I I I}\right) C_{33}^{I I I} \omega_{i}^{I I I} \\
& +C_{13}^{I I I} \xi^{2} \theta_{i}^{I I I}, \\
& \Phi_{i}^{I I}=e^{-\left(\beta^{I I}-\lambda_{i}^{I I}\right) h} \varphi_{i}^{I I}, \\
& \Theta_{i}^{I I}=e^{-\left(\beta^{I I}+\lambda_{i}^{I I}\right) h} \theta_{i}^{I I}, \\
& \Theta_{i}^{I I I}=e^{-\left(\beta^{I I I}-\lambda_{i}^{I I I}\right) h} \theta_{i}^{I I I}, \\
& \Psi_{i}^{I I}=e^{-\left(\beta^{I I}-\lambda_{i}^{I I}\right) h} \vartheta_{i}^{I I}, \\
& \Omega_{i}^{I I}=e^{-\left(\beta^{I I}+\lambda_{i}^{I I}\right) h} \omega_{i}^{I I}, \\
& \Omega_{i}^{I I I}=e^{-\left(\beta^{I I I}+\lambda_{i}^{I I I}\right) h} \omega_{i}^{I I I}, \\
& p_{i}^{I I}=e^{\left(\beta^{I I}+\lambda_{i}^{I I}\right) h} \eta_{i}^{I I}, \\
& q_{i}^{I I}=e^{\left(\beta^{I I}-\lambda_{i}^{I I}\right) h} \xi_{i}^{I I}, \\
& q_{i}^{I I I}=e^{\left(\beta^{I I I}-\lambda_{i}^{I I}\right) h} \xi_{i}^{I I I}, \\
& n_{i}^{I I}=e^{\left(\beta^{I I}+\lambda_{i}^{I I}\right) h} v_{i}^{I I}, \\
& t_{i}^{I I}=e^{\left(\beta^{I I}-\lambda_{i}^{I I}\right) h} \nu_{i}^{I I}, \\
& t_{i}^{I I I}=e^{\left(\beta^{I I I}-\lambda_{i}^{I I I}\right) h} \nu_{i}^{I I I} .
\end{aligned}
$$

\section{Biographies}

Yaser Zafari received his MSc degree from School of Civil Engineering, University of Tehran, Iran, in 2016. He has studied mechanics of composites and inhomogeneous materials through analytical and numerical methods. His research fields are computational mechanics, elastostatics of anisotropic materials and composite structures.

Mehdi Shahmohamadi received his $\mathrm{PhD}$ degree from University of Tehran, Iran, in 2017. He has studied interaction analysis in anisotropic and inhomogeneous materials through semi-analytical methods. He has published 7 papers in ISI indexed journals.

Ali Khojasteh, the current Assistant Professor at the University of Tehran, received his MSc and $\mathrm{PhD}$ degrees from the University of Tehran, Iran, in 2005 and 2009, respectively, both ranked 1st among graduates. His research interests are wave propagation in solids/fluids, computational mechanics, mechanics of composites, boundary element method, and soilstructure Interaction. He was ranked 262nd among more than 800,000 and 7th among more than 15000 participating in the nationwide undergraduate and MSc program entrance exams, respectively. He was also ranked 9th among more than 15000 in the national Civil Engineering Olympiad. He has published more than 20 papers in international ISI indexed journals.

Mohammad Rahimian, currently the Full Professor of Civil Engineering Department at the University of Tehran, received his BSc in Civil Engineering from University of Tehran. He received his $\mathrm{PhD}$ in Ecole National Des Ponts et Chaussees, France, in 1981. His research fields are earthquake engineering, elastodynamics of anisotropic materials, soil-structure-fluid interaction, etc. He is the Assistant to Chair of the University of Tehran and the Former Chair of the University of Tehran. More than 45 papers published in international ISI indexed journals, along with 6 written or translated books are of his scientific works. 